\title{
Berberine inhibits Wilms' tumor cell progression through upregulation of Wilms' tumor gene on the $X$ chromosome
}

\author{
YAN LIU ${ }^{1}$ and SHENG LIU ${ }^{2}$ \\ ${ }^{1}$ Department of Pediatrics, The First Affiliated Hospital of Henan University of Science and Technology, \\ Luoyang, Henan 475000; ${ }^{2}$ Department of Pharmacology, Basic Medical College of Zhengzhou University, \\ Zhengzhou, Henan 450001, P.R. China
}

Received May 9, 2013; Accepted July 31, 2013

DOI: $10.3892 / \mathrm{mmr} .2013 .1665$

\begin{abstract}
Wilms' tumor is a type of kidney cancer that affects young children. Although a number of Wilms' tumor samples have been collected through international trials, the mechanisms underlying its progression remain challenging to determine. Extensive studies have identified somatic mutations at several loci in Wilms' tumorigenesis, including WT1, catenin, Wilms' tumor gene on the X chromosome (WTX) and TP53. Berberine is a benzylisoquinoline alkaloid extracted from numerous types of medicinal plants and has been extensively used as a Chinese traditional medicine. Recently, berberine has been demonstrated to possess antitumoral activities. AMP-activated protein kinase (AMPK) is suggested to be one of the various cellular targets of berberine, which regulates tumor progression and metastasis. However, the specific involvement of berberine-induced AMPK activation and its effects on the proliferation potential of Wilms' tumor cells remains unknown. The present study investigated the berberine-induced activation of AMPK and its effects on G401 Wilms' tumor cell proliferation. The results demonstrated that berberine inhibited growth and decreased the expression of cell-cycle regulators in these cells. At the molecular level, berberine treatment led to a significant increase of WTX expression and G401 cells were protected against berberine-induced growth inhibition by small interfering RNA against WTX. In conclusion, these results suggest a novel mechanism that may contribute to the antineoplastic effects of berberine which was also demonstrated by recent population studies; however, further studies are required to investigate the potential therapeutic use of berberine in patients with Wilms' tumor.
\end{abstract}

Correspondence to: Dr Sheng Liu, Department of Pharmacology, Basic Medical College of Zhengzhou University, Daxue Street, Zhengzhou, Henan 450001, P.R. China

E-mail: yanliu5509@163.com

Key words: berberine, Wilms' tumor, WTX, AMP-activated protein kinase

\section{Introduction}

Wilms' tumor is a type of kidney cancer that affects young children. International trials have been established, which have collected a number of Wilms' tumor samples; however, the mechanisms underlying its pathogenesis and effective treatment strategies remain to be clearly determined (1). Extensive studies have identified somatic mutations at several loci in Wilms' tumorigenesis, including WT1, CTNNB1, TP53 and Wilms' tumor gene on the X chromosome (WTX) $(2,3)$.

WTX has been considered as a key tumor suppressor in Wilms' tumor. Inactivation of WTX is the most frequent genetic event in sporadic Wilms' tumor, reported in up to $30 \%$ of cases (4). It has been suggested that WTX interacts with the anaphase-promoting complex and negatively regulates $\beta$-catenin stability (5). It also modulates the transcriptional activity of WT1, another Wilms' tumor suppressor that encodes a zinc finger transcriptional regulator of cellular differentiation (6). Additionally, previous studies have also indicated a positive effect on 553 signaling through enhancing CBP/P300-mediated acetylation of p53 at Lysine 382 (7). Therefore, WTX regulates Wilms' tumor initiation and progression through several mechanisms.

Berberine, a well-studied naturally occurring isoquinoline alkaloid, is an active component of the Ranunculaceae and Papaveraceae plant families. Recent studies focused on its antitumor effect have shown that berberine inhibits the growth of multiple tumor cell types derived from the liver, lung, gastrointestinal tract, leukocytes, brain, skin, bladder, bone, breast and prostate (8-13). At the molecular level, several mechanisms involved in the antitumor activity of berberine have been identified, including stimulating caspase-dependent apoptosis and caspase-independent cell death by the activation of apoptosis-inducing factors, suppressing tumor cell proliferation and growth by the induction of cell-cycle arrest, and inhibiting metastasis by downregulating matrix metalloproteinases (14-16). Numerous signaling pathways, including $\mathrm{p} 53, \mathrm{NF}-\kappa \mathrm{B}$ and MAPK pathways have been identified to be involved in the anticancer effects of berberine (12,17-19). Therefore, the results suggesting that the mechanisms underlying berberine's anticancer effects are distinct among tumor cell types suggest a cell-type specific effect of berberine on the inhibition of tumor progression. 
However, it remains unclear whether berberine directly inhibits proliferation in the G401 human Wilms' tumor cell line. Thus, the aim of the present study was to investigate the effect of berberine on G401 cell proliferation and the underlying molecular mechanisms, to potentially provide results which may aid in the development of effective drugs for the clinical treatment of Wilms' tumor.

\section{Materials and methods}

Cell cultures. The G401 Wilms' tumor cell line was purchased from the Cell Bank of Type Culture Collection of Chinese Academy of Sciences (Shanghai, China), and cultured in RPMI-1640 (Dulbecco's modified Eagle's medium) supplemented with $10 \%$ fetal calf serum, $100 \mathrm{IU} / \mathrm{ml}$ penicillin and $100 \mathrm{mg} / \mathrm{ml}$ streptomycin (all obtained from Gibco-BRL, Carlsbad, CA, USA). Cultures were maintained at $37^{\circ} \mathrm{C}$ in a humidified $5 \% \mathrm{CO}_{2}$ atmosphere.

Cell viability and bromodeoxyuridine (BrdU) incorporation assays. Cell viability was measured by the 3-(4,5-dimethylthiazol-2-yl)-2,5-diphenyltetrazolium bromide (MTT) assays using kits from Sigma-Aldrich, St. Louis, MO, USA. MTT assays were performed by incubating the cells with $0.4 \mathrm{mg} / \mathrm{ml}$ MTT for $6 \mathrm{~h}$. The formazan product was dissolved in dimethyl sulfoxide, and absorbance was read at $490 \mathrm{~nm}$. A cell proliferation enzyme-linked immunosorbent assay kit (Beyotime, Shanghai, China) was used to measure the incorporation of BrdU during DNA synthesis according to the manufacturer's instructions. All experiments were repeated at least four times in triplicate.

RNA isolation and $q P C R$. Total RNA was isolated from cells by TRIzol reagent (Invitrogen Life Technologies, Carlsbad, CA, USA), and reverse transcription was conducted using a Takara RNA PCR kit (Takara Biotechnology, Dalian, China), according to the manufacturer's instructions. In order to analyze the transcripts of the genes of interest, qPCR was performed using an SYBR-Green Premix Ex Taq (Takara Biotechnology) on an ABI 7500 machine (Invitrogen Life Technologies).

Western blot analysis. Cells were harvested and lysed with ice-cold lysis buffer (50 mM Tris-HCl, pH 7.4; 100 mM DTT; $2 \% \mathrm{w} / \mathrm{v} \mathrm{SDS}$; and $10 \%$ glycerol). Following centrifugation at $20,000 \mathrm{x} \mathrm{g}$ for $10 \mathrm{~min}$ at $4^{\circ} \mathrm{C}$, proteins in the supernatants were quantified and separated by $10 \%$ sodium dodecyl sulfate-polyacrylamide gel electrophoresis, and transferred onto nitrocellulose membranes. Subsequent to blocking with 5\% non-fat milk, membranes were immunoblotted with antibodies, followed by horseradish peroxidase (HRP)-linked secondary antibodies. The signals were detected by Millipore SuperSignal ${ }^{\circledR}$ HRP Substrate kit (Millipore, Billerica, MA, USA) according to the manufacturer's instructions. Anti-glyceraldehyde 3-phosphate dehydrogenase (GAPDH), anti-AMP-activated protein kinase (AMPK), anti-ACC, anti-MAPK, anti-S6K and anti-WTX antibodies were purchased from Santa Cruz Biotechnology, Inc. (Santa Cruz, CA, USA) or and anti-p21, anti-p27 and anti-cyclin E antibodies were obtained from Cell Signaling Technology Inc. (Danvers, MA, USA).
Small interfering RNA (siRNA). Cells were transfected with siRNA targeting the WTX or luciferase gene (all siRNA oligos from Qiagen, Valencia, CA, USA) using Lipofectamine 2000 (Invitrogen Life Technologies) according to the manufacturer's instructions. Cell cultures were incubated for $18 \mathrm{~h}$ with $100 \mathrm{nM}$ siRNA prior to berberine treatment.

Statistical analysis. Statistical analysis was performed with a paired Student's t-test or two-way analysis of variance test. Numerical data are presented as the mean \pm SEM. ${ }^{*} \mathrm{P}<0.05$, ${ }^{* *} \mathrm{P}<0.01$ or ${ }^{* * *} \mathrm{P}<0.001$ were considered to indicate a statistically significant difference.

\section{Results}

Berberine treatment inhibits cell growth in a dose-dependent manner. To the best of our knowledge, the effects of berberine on Wilms' tumor cells has not been previously analyzed. Thus, G401 cells were selected to investigate whether berberine exhibits potential anti-proliferative functions. G401 cells were treated with berberine at several concentrations. After $48 \mathrm{~h}$ of treatment, growth was inhibited in a dose-dependent manner as determined by MTT and BrdU incorporation assays (Fig. 1). Moreover, these results suggested that the concentration of berberine at $20 \mu \mathrm{M}$ was optimal in G401 lines. Therefore, $20 \mu \mathrm{M}$ of berberine was selected for the further analysis of gene expression in G401 cells.

Expression of $p 27, p 21$ and cyclin $E$ in berberine-treated cells. It was speculated that growth inhibition in G401 cells may be due to cell-cycle arrest following berberine treatment. To confirm this hypothesis, the expression of p21, p27 and cyclin E was analyzed, which are known to be key molecules in cell-cycle arrest. Expression levels of p21 and p27 were significantly increased in G401 cells (Fig. 2). In addition, the contents of cyclin $\mathrm{E}$ were markedly downregulated in berberine-treated cells (Fig. 2).

Berberine upregulates AMP kinase activity in Wilms' tumor cells. Several studies have indicated that the antiproliferative effects of berberine involve the AMP kinase pathway (19). In the present study, the results of the western blot analysis indicated that berberine stimulated AMPK phosphorylation in G401 cells (Fig. 3A). Phosphorylated ACC, a downstream target of AMPK, was also enhanced in cells treated with berberine (Fig. 3A). As AMPK activation inhibits energy-consuming pathways and protein synthesis, it was observed that AMPK activation is associated with an increase in the phosphorylation of mTOR and S6 kinase (Fig. 3B).

Berberine increases WTX expression in G401 cells. The expression of several cell-cycle regulators, including $\mathrm{p} 21$, is controlled by tumor suppressor WTX (20). As these genes are regulated by berberine treatment in G401 cells, WTX abundance was analyzed in these cells. It was observed that WTX expression was markedly increased following berberine treatment in G401 cells (Fig. 4).

siRNA against WTX rescues cells from berberine-induced growth inhibition. To determine whether the induction of 
A

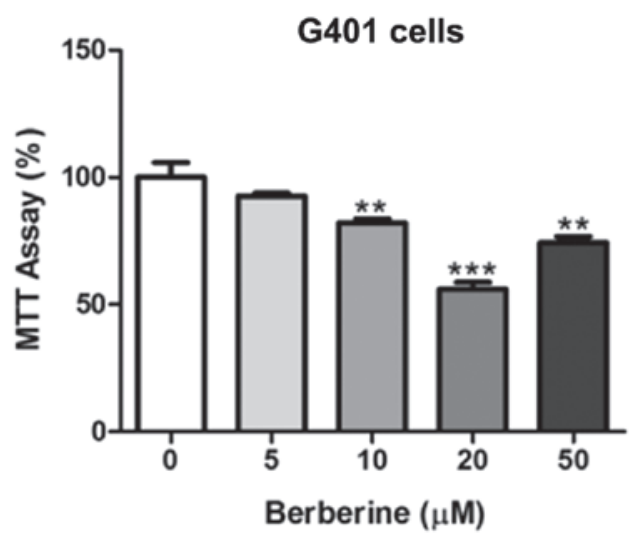

B

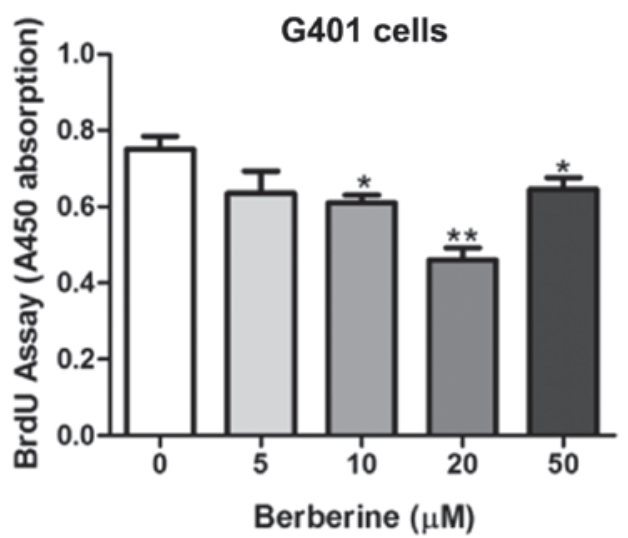

Figure 1. Berberine inhibits cell proliferation in Wilms' tumor cells. (A) Cell viability was measured by MTT assays in G401 cells. Cells were treated with the indicated concentrations of berberine for $48 \mathrm{~h}$. (B) Cell proliferation activity was measured by BrdU incorporation assays in G401 cells. BrdU, bromodeoxyuridine. ${ }^{*} \mathrm{P}<0.05 ;{ }^{* *} \mathrm{P}<0.01 ;{ }^{* * *} \mathrm{P}<0.001$.

A

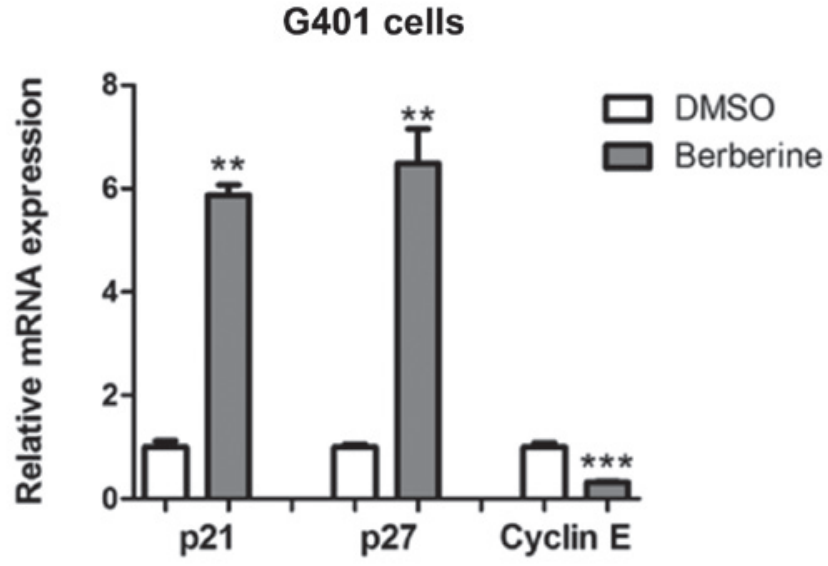

B DMSO Berberine

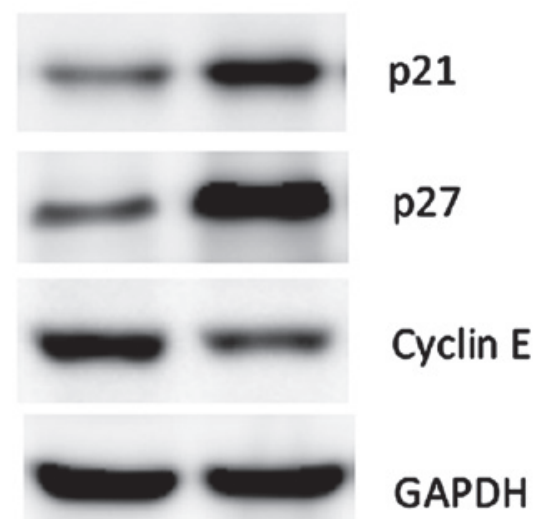

Figure 2. Berberine regulates the expression of cell-cycle regulators. (A) mRNA and (B) protein levels of p21, p27 and cyclin E were determined by qPCR and western blot analysis in G401 cells treated with vehicle control (DMSO) or berberine (20 $\mu \mathrm{M})$. DMSO, dimethyl sulfoxide. GAPDH was used as a loading control. ${ }^{* *} \mathrm{P}<0.01 ;{ }^{* * * *} \mathrm{P}<0.001$.

A
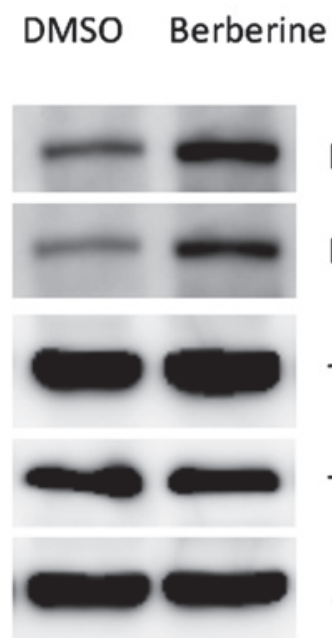

P-AMPK

P-ACC

T-AMPK

T-ACC

GAPDH
B

DMSO Berberine

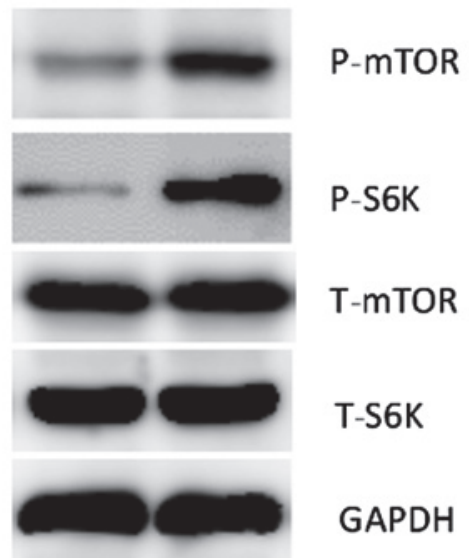

Figure 3. Berberine induces AMPK activation in Wilms' tumor cells. (A) Western blot analysis of phosphorylated AMPK and ACC (P-AMPK and P-ACC) in G401 cells. Contents of total AMPK, ACC (T-AMPK and T-ACC) and GAPDH were used as loading controls. (B) Western blot analysis of phosphorylated mTOR and S6K (P-mTOR and P-S6K) in G401 cells. Contents of total mTOR, S6K (T-mTOR and T-S6K) and GAPDH were used as loading controls. AMPK, AMP-activated protein kinase; GAPDH, glyceraldehyde 3-phosphate dehydrogenase. 
A

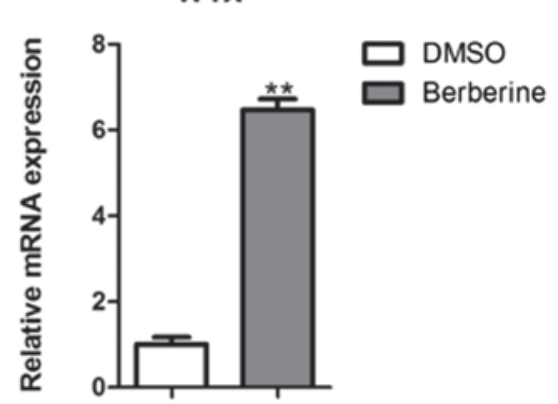

B

\section{DMSO Berberine}

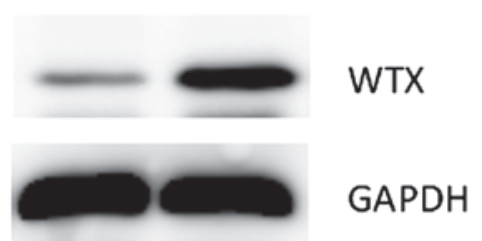

Figure 4. Berberine increased WTX expression in Wilms' tumor cells. (A) mRNA and (B) protein levels of WTX in G401 cells treated with vehicle control (DMSO) or berberine. DMSO, dimethylsulfoxide ( $\left.{ }^{* *} \mathrm{P}<0.01\right)$

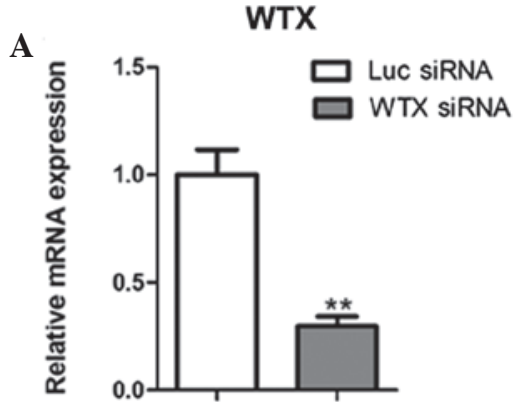

D

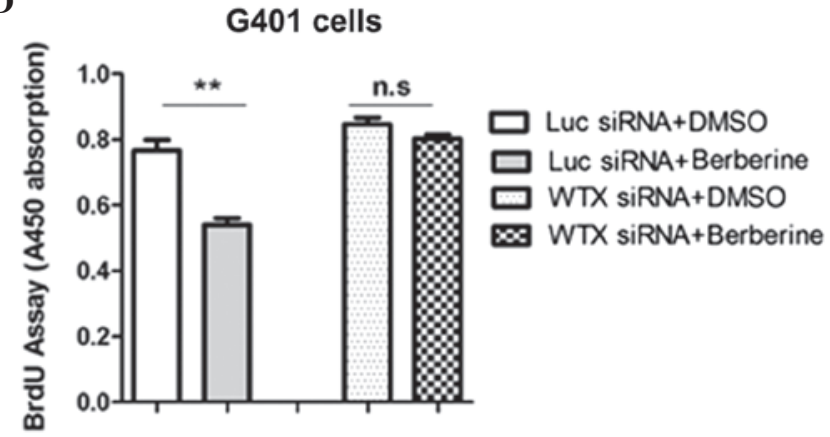

B SIRNA LUC WTX

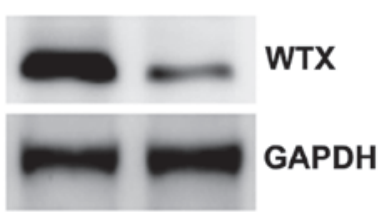

$\mathbf{E}$
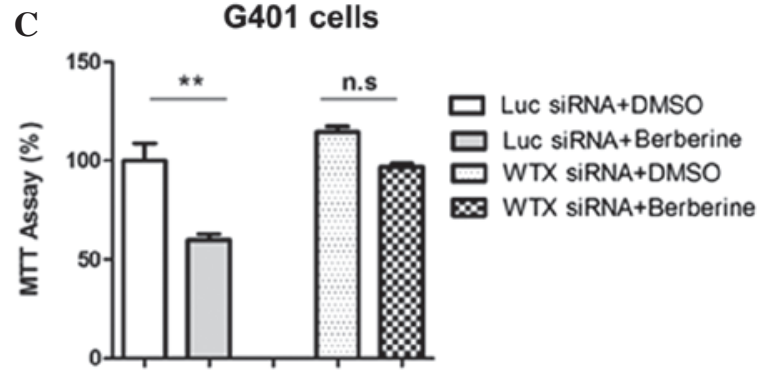

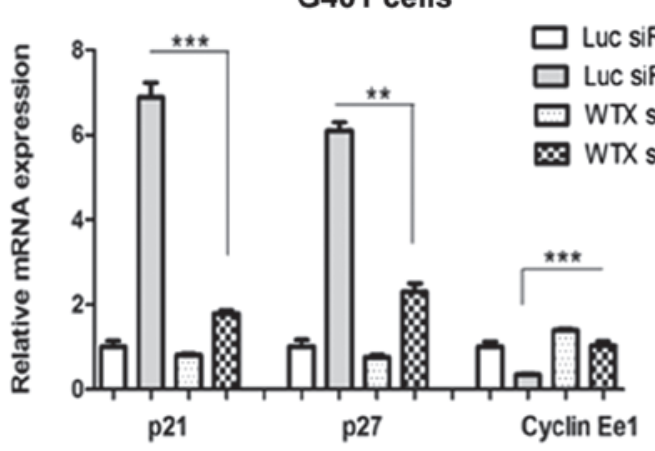

Figure 5. WTX deficiency reversed the anti-proliferative involvement of berberine. (A) mRNA and (B) protein levels of WTX in G401 cells transfected with small inhibitory (si)RNA oligos against WTX that had been treated with berberine or vehicle control (DMSO). siRNA oligos targeting Luc were used as a negative control. (C) Cell viability and (D) proliferation activity were measured by MTT and BrdU assays in G401 cells. (E) mRNA levels of p21, p27 and cyclin E were determined by qPCR in G401 cells. DMSO, dimethylsulfoxide; Luc, luciferase; BrdU, bromodeoxyuridine. ${ }^{* *} \mathrm{P}<0.01 ;{ }^{* * * *} \mathrm{P}<0.001 ; \mathrm{n} . \mathrm{s}$, no significance.

WTX by berberine is required for the anti-proliferative effect of the drug, WTX knockdown experiments using siRNA oligos were conducted(Fig. 5A and B). As a result, the siRNA rescued G401 cells from the inhibitory effect of berberine (Fig. 5C and D). Consistently, the inhibitory functions of berberine on the expression levels of cell-cycle regulators were also reversed by WTX siRNA oligos (Fig. 5E). Therefore, the results indicate WTX as a potential novel molecular target in anticancer therapy, which is upregulated by berberine treatment.

\section{Discussion}

In the present study, the involvement of berberine and its molecular mechanism in Wilms' tumor cells was investigated. Berberine was shown to inhibit cell proliferation in G401 cells as demonstrated by MTT and BrdU incorporation assays. Moreover, berberine treatment induced p21 and p27 expression while repressing cyclin E expression. At the molecular level, the results demonstrated that berberine activated AMP kinase activation and inhibited mTOR signaling. In addition, WTX was identified to be a novel molecular target of berberine. WTX invalidation, using siRNA oligos, abrogated berberine inhibition in G401 tumor cells. In conclusion, the data suggested that berberine may be beneficial in the treatment of Wilms' tumor.

Previous studies have suggested that the antiproliferative effects of berberine involved the AMP kinase pathway. In the present study, inhibition of AMPK signaling reversed the anticancer roles of berberine. AMPK is a highly conserved Ser/Thr protein kinase complex that is central in the regulation of cellular energy homeostasis (21). AMPK is activated 
in response to decreased fuel supply and functions in the allocation of nutrients toward catabolic/energy-producing or anabolic/growth-promoting metabolic pathways (22). From a metabolic standpoint, AMPK promotes ATP conservation under conditions of metabolic stress by activating pathways of catabolic metabolism (such as autophagy) and inhibiting anabolic processes (including lipid biosynthesis, mTOR-dependent protein synthesis, cell growth and proliferation) $(23,24)$. Thus, AMPK activity has been associated with stress resistance and survival in tumor cells. Moreover, AMPK activation was shown to be associated with certain tumor suppressors, including the p53 pathway. AMPK activation induces phosphorylation of p53 on serine 15, and this phosphorylation is suggested to be required to initiate AMPK-dependent cell-cycle arrest (25). Consistently, AMPK-induced p53 activation promotes cellular survival in response to glucose deprivation, and cells that have undergone a p53-dependent metabolic arrest rapidly re-enter the cell cycle upon glucose restoration. Moreover, persistent activation of AMPK leads to accelerated p53-dependent cellular senescence (25). Notably, this study demonstrated that berberine activates AMPK signaling and simultaneously induces WTX expression in G401 cells. Therefore, further investigation is required to determine whether AMPK regulates WTX at the transcriptional or post-transcriptional levels.

In conclusion, the results suggest the underlying mechanisms that may contribute to the antineoplastic effects of berberine. Further studies are required to investigate the potential of berberine as a therapy for Wilms' tumor prevention and treatment.

\section{References}

1. Scott RH, Stiller CA, Walker L and Rahman N: Syndromes and constitutional chromosomal abnormalities associated with Wilms tumour. J Med Genet 43: 705-715, 2006.

2. Malkin D, Sexsmith E, Yeger H, Williams BR and Coppes MJ: Mutations of the p53 tumor suppressor gene occur infrequently in Wilms' tumor. Cancer Res 54: 2077-2079, 1994.

3. Grundy PE, Breslow NE, Li S, et al; National Wilms Tumor Study Group: Loss of heterozygosity for chromosomes 1p and $16 \mathrm{q}$ is an adverse prognostic factor in favorable-histology Wilms tumor: a report from the National Wilms Tumor Study Group. J Clin Oncol 23: 7312-7321, 2005.

4. Rivera MN, Kim WJ, Wells J, et al: An X chromosome gene, WTX, is commonly inactivated in Wilms tumor. Science 315 642-645, 2007.

5. Major MB, Camp ND, Berndt JD, et al: Wilms tumor suppressor WTX negatively regulates $\mathrm{WNT}$ /beta-catenin signaling. Science 316: 1043-1046, 2007

6. Rivera MN and Haber DA: Wilms' tumour: connecting tumorigenesis and organ development in the kidney. Nat Rev Cancer 5: 699-712, 2005.
7. Kim WJ, Rivera MN, Coffman EJ and Haber DA: The WTX tumor suppressor enhances p53 acetylation 9 by $\mathrm{CBP} / \mathrm{p} 300$. Mol Cell 45: 587-597, 2012.

8. Hou Q, Tang X, Liu H, et al: Berberine induces cell death in human hepatoma cells in vitro by downregulating CD147. Cancer Sci 102: 1287-1292, 2011.

9. James MA, Fu H, Liu Y, Chen DR and You M: Dietary administration of berberine or Phellodendron amurense extract inhibits cell cycle progression and lung tumorigenesis. Mol Carcinog 50: $1-7,2011$.

10. Harikumar KB, Kuttan G and Kuttan R: Inhibition of progression of erythroleukemia induced by Friend virus in BALB/c mice by natural products - berberine, curcumin and picroliv. J Exp Ther Oncol 7: 275-284, 2008

11. Yan K, Zhang C, Feng J, et al: Induction of G1 cell cycle arrest and apoptosis by berberine in bladder cancer cells. Eur J Pharmacol 661: 1-7, 2011.

12. Kim S, Han J, Kim NY, et al: Effect of berberine on $\mathrm{p} 53$ expression by TPA in breast cancer cells. Oncol Rep 27: 210-215, 2012.

13. Meeran SM, Katiyar S and Katiyar SK: Berberine-induced apoptosis in human prostate cancer cells is initiated by reactive oxygen species generation. Toxicol Appl Pharmacol 229: 33-43, 2008.

14. Lv X, Yu X, Wang Y, et al: Berberine inhibits doxorubicin-triggered cardiomyocyte apoptosis via attenuating mitochondrial dysfunction and increasing Bcl-2 expression. PLoS One 7: e47351, 2012.

15. Tillhon M, Guamán Ortiz LM, Lombardi P and Scovassi AI: Berberine: new perspectives for old remedies. Biochem Pharmacol 84: 1260-1267, 2012.

16. Kim S, Han J,Lee SK, et al: Berberine suppresses the TPA-induced MMP-1 and MMP-9 expressions through the inhibition of PKC- $\alpha$ in breast cancer cells. J Surg Res 176: e21-e29, 2012.

17. Chitra P, Saiprasad G, Manikandan R and Sudhandiran G: Berberine attenuates bleomycin induced pulmonary toxicity and fibrosis via suppressing NF- $\kappa \mathrm{B}$ dependent TGF- $\beta$ activation: a biphasic experimental study. Toxicol Lett 219: 178-193, 2013.

18. Alzamora R, O'Mahony F, Ko WH, Yip TW, Carter D, Irnaten M and Harvey BJ: Berberine reduces cAMP-induced chloride secretion in T84 human colonic carcinoma cells through inhibition of basolateral KCNQ1 channels. Front Physiol 2: 33, 2011.

19. Kim HS, Kim MJ, Kim EJ, Yang Y, Lee MS and Lim JS: Berberineinduced AMPK activation inhibits the metastatic potential of melanoma cells via reduction of ERK activity and COX-2 protein expression. Biochem Pharmacol 83: 385-394, 2012.

20. Kim MK, Min DJ, Rabin M and Licht JD: Functional characterization of Wilms tumor-suppressor WTX and tumor-associated mutants. Oncogene 30: 832-842, 2011.

21. Hardie DG: AMP-activated protein kinase: an energy sensor that regulates all aspects of cell function. Genes Dev 25: 1895-1908, 2011.

22. Egan DF, Shackelford DB, Mihaylova MM, et al: Phosphorylation of ULK1 (hATG1) by AMP-activated protein kinase connects energy sensing to mitophagy. Science 331: 456-461, 2011.

23. Gwinn DM, Shackelford DB, Egan DF, et al: AMPK phosphorylation of raptor mediates a metabolic checkpoint. Mol Cell 30: 214-226, 2008

24. Liu L, Ulbrich J, Müller J, et al: Deregulated MYC expression induces dependence upon AMPK-related kinase 5. Nature 483: 608-612, 2012

25. Jones RG, Plas DR, Kubek S, et al: AMP-activated protein kinase induces a p53-dependent metabolic checkpoint. Mol Cell 18: 283-293, 2005. 